\title{
MULTISCALE SEGMENTATION OF POLARIMETRIC SAR IMAGE BASED ON SRM SUPERPIXELS
}

\author{
F. Lang ${ }^{\mathrm{a}, *}$, J. Yang ${ }^{\mathrm{b}}, \mathrm{L} . \mathrm{Wu}^{\mathrm{a}}, \mathrm{D} . \mathrm{Li}^{\mathrm{b}}$, a \\ ${ }^{a}$ School of Environment Science and Spatial Informatics, China University of Mining and Technology, No.1, Daxue Road, Xuzhou \\ 221116, China - *langfk@cumt.edu.cn \\ ${ }^{\mathrm{b}}$ State Key Laboratory of Information Engineering in Surveying, Mapping and Remote Sensing, Wuhan University, 129 Luoyu \\ Road, Wuhan, 430079, China
}

Commission VII, WG VII/4

KEY WORDS: synthetic aperture radar (SAR), polarimetric SAR (PolSAR), segmentation, multiscale, superpixel, binary partition tree (BPT), statistical region merging (SRM)

\begin{abstract}
:
Multi-scale segmentation of remote sensing image is more systematic and more convenient for the object-oriented image analysis compared to single-scale segmentation. However, the existing pixel-based polarimetric SAR (PolSAR) image multi-scale segmentation algorithms are usually inefficient and impractical. In this paper, we proposed a superpixel-based binary partition tree (BPT) segmentation algorithm by combining the generalized statistical region merging (GSRM) algorithm and the BPT algorithm. First, superpixels are obtained by setting a maximum region number threshold to GSRM. Then, the region merging process of the BPT algorithm is implemented based on superpixels but not pixels. The proposed algorithm inherits the advantages of both GSRM and BPT. The operation efficiency is obviously improved compared to the pixel-based BPT segmentation. Experiments using the Lband ESAR image over the Oberpfaffenhofen test site proved the effectiveness of the proposed method.
\end{abstract}

\section{INTRODUCTION}

Image segmentation is an important pre-processing step in the object-based image analysis (OBIA) technology. In the use of single-scale segmentation algorithm for image segmentation, it is often required to segment a few times, and then choose a best segmentation result. On the one hand, the efficiency of this approach is low due to multiple segmentations. On the other hand, the final segmentation result is usually not optimal since it is chosen from limited number of results. In order to overcome the defects of single-scale segmentation, a number of multi-scale segmentation algorithms have been proposed, such as quadtrees (Samet, 1985), pyramid (Jolion and Montanvert, 1992; Koepfler et al., 1994), and binary partition trees (BPT) (Salembier, 2000). Inspired by the scale space theory (Witkin, 1983; Koenderink, 1984), Guigues et al. (2003) proposed a scale-sets image analysis method by putting these multi-scale image representation algorithms into the framework of scalesets theory. Compared with the single-scale segmentation, the multi-scale segmentation can obtain multi-scale segmentation results which constitute a complete hierarchical structure on different scales by only once segmentation. Therefore, multiscale segmentation is more systematic and more convenient for OBIA.

Recently, Alonso-Gonzndezlez et al. (2010, 2011, 2012) proposed a polarimetric SAR (PolSAR) image BPT segmentation algorithm. This algorithm has good potential in OBIA since it can keep image details on small scale as well as reveal the contour information of large homogeneous regions on large scale. However, this algorithm is inefficient and can be easily affected by the speckle noise since the BPT is built on single pixels. In this paper, we proposed a multiscale segmentation algorithm by combing the BPT segmentation and a superpixel segmentation algorithm.

\section{GSRM SUPERPIXEL}

Statistical region merging (SRM) is a segmentation algorithm proposed by Nock and Nielsen (2004). It was originally used only for the field of pattern recognition. Later, Li et al. (2008) introduced it into object-oriented classification of PolSAR image. Lang et al. $(2012$, 2014) proposed a generalized SRM (GSRM) segmentation algorithm which was more suitable for object-based classification of PolSAR image. Compared with the existing segmentation algorithms, the GSRM segmentation algorithm does not depend on the probability distribution of the data, has high efficiency, and has a good performance in coping with noise corruption. These advantages make it very suitable for PolSAR image segmentation.

GSRM belongs to region growing and merging technology, which mainly consists of two elements: the merging criterion confirming whether adjacent regions are merged or not, and the merging order followed to test the merging of regions.

Suppose $I_{G}$ is an observed image that contains $N_{I}$ pixels. Each pixel in $I_{G}$ contains a non-negative real value in each channel. The noise in $I_{G}$ is multiplicative. Consider a pair of adjacent regions $\left(R, R^{\prime}\right)$ in $I_{G}$, the merging criterion is:

$$
P_{G}\left(R, R^{\prime}\right)=\left\{\begin{array}{lc}
\text { true. } & \left\|\overline{R^{\prime}}-\bar{R}\right\|_{1} \leq b_{G}\left(R, R^{\prime}\right) \\
\text { false. } & \text { otherwise }
\end{array}\right.
$$


where

$$
b_{G}\left(R, R^{\prime}\right)=\sqrt{\frac{B^{2}}{2 Q}\left(\frac{\|\mathrm{E}(\bar{R})\|_{1}^{2}}{N_{R}}+\frac{\left\|\mathrm{E}\left(\overline{R^{\prime}}\right)\right\|_{1}^{2}}{N_{R^{\prime}}}\right) \ln \frac{2}{\delta}}
$$

and

$$
\begin{aligned}
& B=\text { normalized constant, usually set } B=2 \\
& Q=\text { segmentation scale adjusting parameter } \\
& \delta=\text { false alarm rate, usually set } \delta=1 /(6 \mathrm{e} 4|I|) \\
& N_{R}, N_{R^{\prime}}=\text { number of pixels in region } R \text { and } R
\end{aligned}
$$

An observed image $I_{G}$ contains $n<4 N_{I}$ pairs of adjacent pixels in 8 -neighborhood. Let $S_{I}$ be the set of these pairs, and $f$ (p,, $\mathbf{p}$ ') be a real-valued gradient function, with $\mathbf{p}$ and $\mathbf{p}$ ' as a pair of adjacent pixels in $I_{G}$. Instead of stepwise optimization tactics, GSRM adopts a pre-ordering strategy. With this strategy, the GSRM algorithm can be described as follows: first, sort the pairs of $S_{I}$ in increasing order of $f\left(\mathbf{p}, \mathbf{p}^{\prime}\right)$, and then traverse this order only once. For any current pair of pixels $\left(\mathbf{p}, \mathbf{p}^{\prime}\right) \in S_{I}$, if $R(\mathbf{p}) \neq R^{\prime}\left(\mathbf{p}^{\prime}\right)$, where $R(\mathbf{p})$ stands for the region to which $\mathbf{p}$ belongs, make the test $P_{G}\left(R(\mathbf{p}), R^{\prime}\left(\mathbf{p}^{\prime}\right)\right)$, and merge $R$ and $R^{\prime}$ if the result is true.

The gradient function defined in GSRM is:

$$
f\left(\mathbf{p}, \mathbf{p}^{\prime}\right)=\left\|\frac{\mathbf{p}-\mathbf{p}^{\prime}}{\mathbf{p}+\mathbf{p}^{\prime}}\right\|_{1}
$$

Usually GSRM will get big regions in homogeneous area. To obtain superpixels, a threshold of maximum region pixels is needed in the region merging process.

\section{BPT SEGMENTATION}

BPT is an image presentation method proposed by Salembier and Garrido (2000). This method expresses and stores images in a region-based and multi-scale form, making the image processing algorithms more simple and efficient. AlonsoGonzález et al. (2010, 2011, 2012) introduced BPT into PolSAR image processing field for filtering and segmentation.

The BPT algorithm consists of two main steps: tree constructing and tree pruning.

In order to construct a BPT, there are two main approaches: 1) top-down approach, which splits the whole image into pixels by dividing a region into two adjacent and mutually disjoint regions in each process step; 2) bottom-up approach, which merges two neighboring pixels or regions into one region in each process step until the whole image is merged into one region. Since the number of possible new splitting schemes may be huge, the bottom-up approach is usually used to construct a BPT.

The bottom-up approach also belongs to the region growing and merging technology. Therefore, the two elements: the merging criterion and the merging order must be defined in order to build a BPT completely. The merging order in BPT algorithm is generally fixed: merging the two adjacent regions whose similarity is maximum in each step. The merging criterion has different definitions according to the different data types. In order to make full use of polarimetric information, the merging criterion is defined as the symmetric revised Wishart distance (Alonso-González et al., 2012) in this paper:

$$
d_{R W}\left(R, R^{\prime}\right)=\left(\operatorname{tr}\left(\mathbf{Z}_{R}^{-1} \mathbf{Z}_{R^{\prime}}\right)+\operatorname{tr}\left(\mathbf{Z}_{R^{\prime}}{ }^{-1} \mathbf{Z}_{R}\right)\right)\left(N_{R}+N_{R^{\prime}}\right)
$$

where $\quad \mathbf{Z}_{R}, \mathbf{Z}_{R}$, = average covariance or coherent matrix of region $R$ and $R^{\prime}$

After building a BPT, a tree pruning step is needed in order to show the information of different levels in the image. Usually there are two tree pruning methods:

1) Pruning based on the number of regions (NoR): From the process of the BPT construction, it is easy to know that every time two regions are merged, the total number of regions should be reduced by 1 , and the number of levels plus 1 . Therefore, if we set the number of required segmented regions, we can easily find the corresponding level. This is the simplest and most direct tree pruning strategy, which is efficient and can control the number of segmented regions.

2) Pruning based on the region homogeneity: This method needs to define a region homogeneity measurement first, and then set a homogeneity threshold. From the merging order of the BPT algorithm, we can deduce that the closer to the root of the BPT, the lower the homogeneity of the region. Accordingly, the tree pruning process can use a top-down strategy that starts from the root node and finds all the father nodes whose homogeneities are less than the threshold. The region homogeneity measurement is defined as:

$$
\begin{aligned}
\phi(R) & =\ln \left(\frac{1}{N_{R}} \sum_{i=1}^{N_{R}} \frac{\left\|\mathbf{Z}^{i}-\mathbf{Z}_{R}\right\|_{F}^{2}}{\left\|\mathbf{Z}_{R}\right\|_{F}^{2}}\right) \\
& =\ln \left(\frac{1}{N_{R}\left\|\mathbf{Z}_{R}\right\|_{F}^{2}} \sum_{i=1}^{N_{R}}\left\|\mathbf{Z}^{i}-\mathbf{Z}_{R}\right\|_{F}^{2}\right.
\end{aligned}
$$

where $\quad \mathbf{Z}^{i}=$ the coherence matrix of the $i$ th pixel in region $R$

\section{SUPERPIXEL-BASED BPT}

In this paper, the region merging process of the BPT algorithm is implemented based on superpixels but not pixels. The procedure of the superpixel-based BPT algorithm is as follows:

Algorithm 1. BPT segmentation based on GSRM superpixels (GSRM-BPT) for PolSAR image

1. Run the GSRM superpixel segmentation algorithm for the PolSAR image.

2. Take each superpixel as a leaf node, calculate the number of total superpixels, and find the adjacent regions of each superpixel.

3. Compute the distances of all adjacent regions according to Eq. (4). Suppose the set of these distances is $\mathbf{S}_{D}$.

4. Find the minimum distance $D_{\min }$ in $\mathbf{S}_{D}$, and merge the two adjacent regions $A$ and $B$ corresponding to $D_{\min }$. Suppose the merged region is $C$.

5. Delete all the distances associated with $\mathrm{A}$ and $\mathrm{B}$ in set $\mathbf{S}_{D}$. Find the adjacent regions of region $\mathrm{C}$, and compute their distances according to Eq. (4). Add the results to $\mathbf{S}_{D}$.

6. Return to Step 4, until $\mathbf{S}_{D}$ is empty.

7. Tree pruning based on NoR or region homogeneity. 


\section{EXPERIMENTS AND ANALYSES}

\subsection{Experimental Data}

The ESAR L-band data set that covers the Oberpfaffenhofen test site is used to evaluate the effectiveness of the proposed algorithm. The original size is $2816 \times 1540$ pixels. To facilitate visual interpretation and evaluation, a preprocessing of 6-look in azimuth and 3-look in range were performed to equalize the resolutions of azimuth and range. The Pauli-RGB image is shown as Figure 1, from which we can see that the test site contains lots of objects, including points, lines, and areas, which are very useful for evaluation.

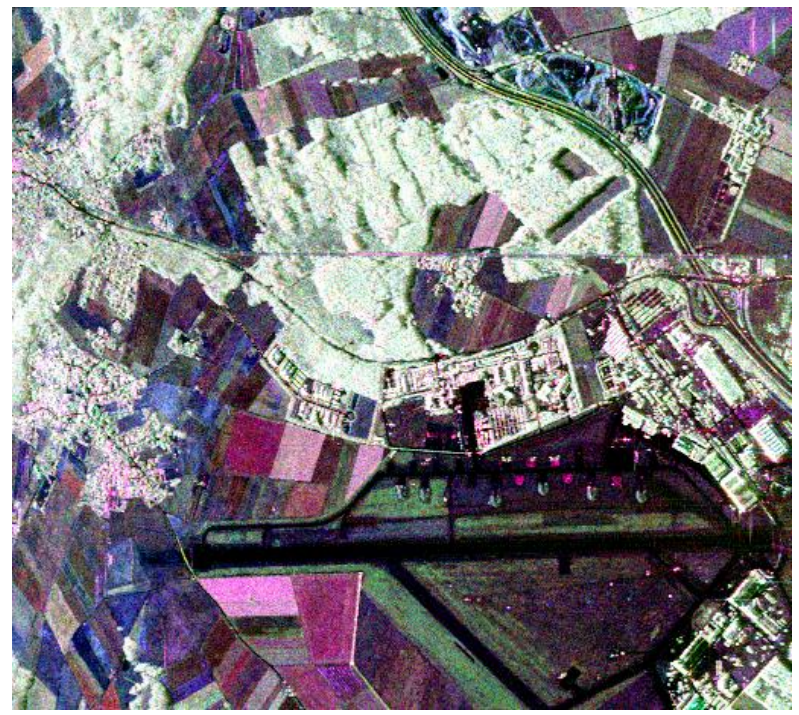

Figure 1. Pauli-RGB image of the ESAR L-band data

\subsection{Evaluation of GSRM Superpixel Segmentation}

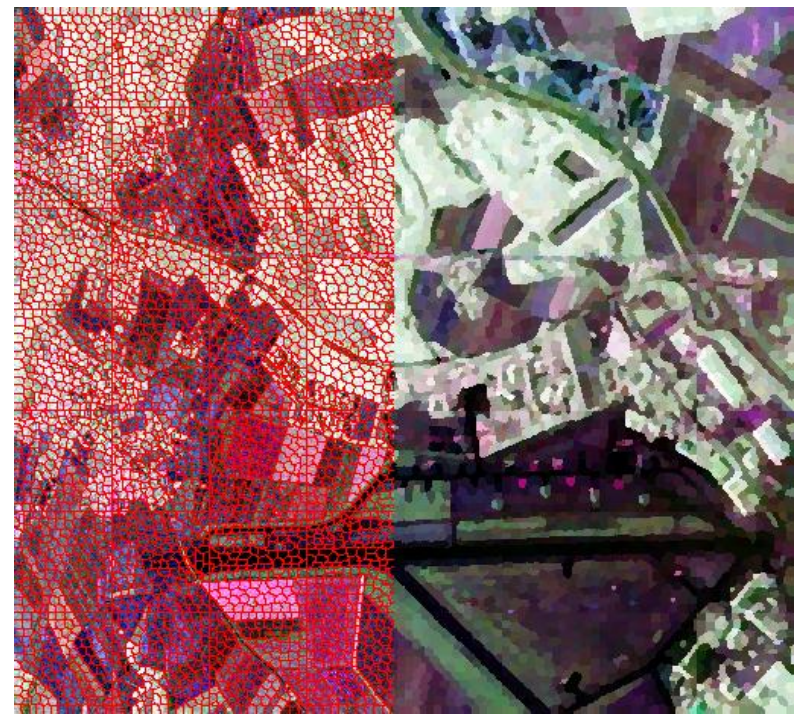

Figure 2. Ncut superpixel segmentation result

The normalized cuts (Ncut) algorithm (Shi and Malik, 2000; Liu et al., 2013) and the GSRM algorithm are applied to the ESAR data for superpixel segmentation respectively. The results are shown in Figure 2 and Figure 3. The NoR in Figure 2 is 8008 . The NoR in Figure 3 is 8166 . From the left side of Figure 2, we can see that the shapes of the segmented regions are regular, and the sizes are similar. But from the right side, we can see that the detail information such as points and lines is lost obviously. From Figure 3, we can see that the shapes of the segmented regions are irregular and the sizes are different. However, the points and lines are preserved very well.

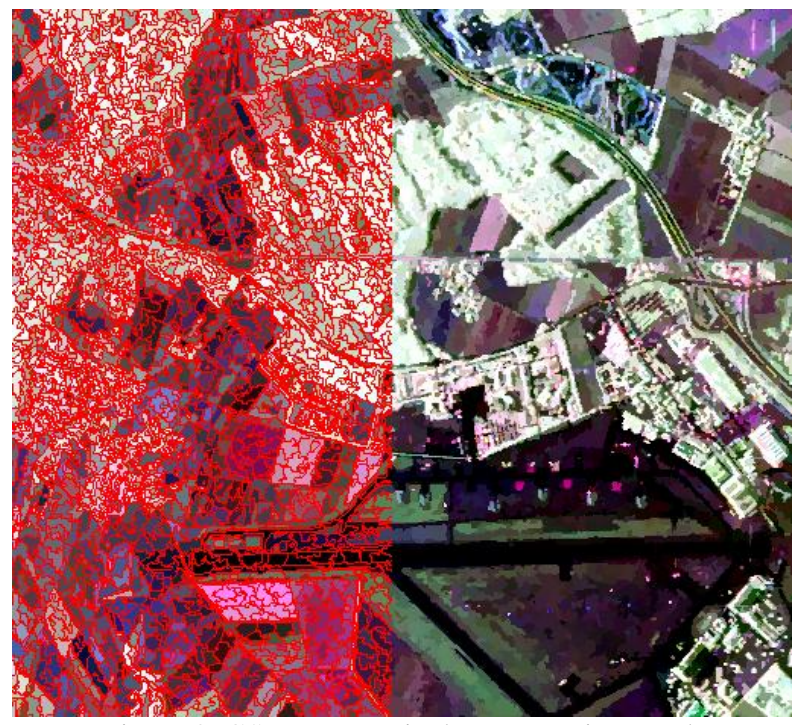

Figure 3. GSRM superpixel segmentation result

\subsection{Evaluation of GSRM-BPT Multiscale Segmentation}

The pixel-based BPT (P-BPT) algorithm and the GSRM-BPT algorithm are applied to the ESAR data for multiscale segmentation respectively. The NoR obtained by GSRM superpixel segmentation algorithm is 14352 . First, the NoRbased pruning strategy is adopted. When the NoR is set 6000 , the results are shown in Figure 4 and Figure 5, from which we can see that the results are very similar. However, under the same condition, the total execution time of P-BPT is about 827 $\mathrm{s}$, and the total execution time of GSRM-BPT is about $176 \mathrm{~s}$. From Table 1, we can see that the majority of the time in BPT algorithm is spent on tree constructing step. The GSRM-BPT algorithm greatly reduces the execution time by constructing BPT based on superpixels but not pixels, since the number of superpixels is much less than the number of pixels.

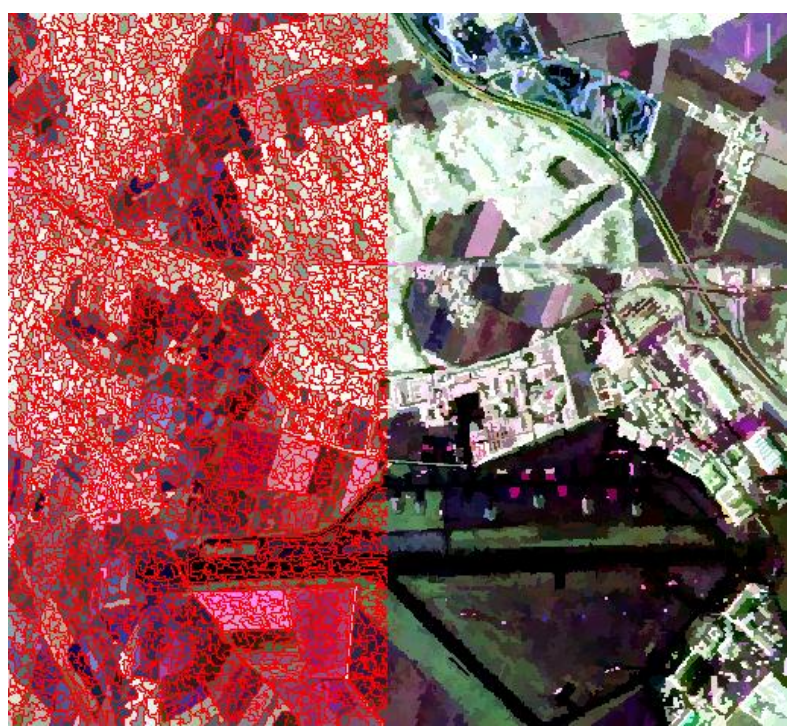

Figure 4. Segmentation result of P-BPT using the NoR-based pruning strategy 


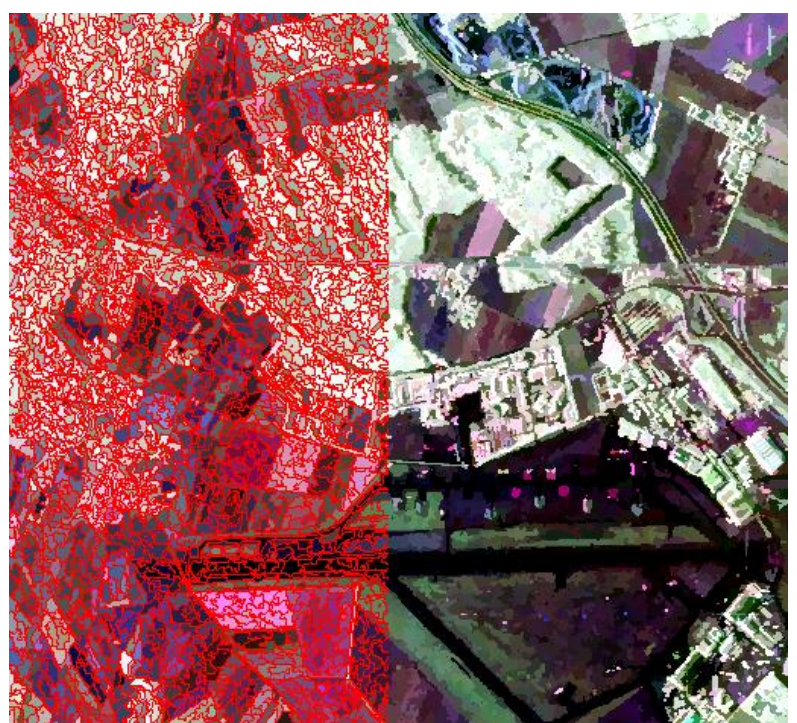

Figure 5. Segmentation result of GSRM-BPT using the NoRbased pruning strategy

\begin{tabular}{|c|c|c|c|}
\hline \multicolumn{2}{|c|}{ Algorithm } & P-BPT & GSRM-BPT \\
\hline \multicolumn{2}{|c|}{ Superpixel segmentation (s) } & - & 3 \\
\hline \multicolumn{2}{|c|}{ Tree constructing(s) } & 826 & 172 \\
\hline \multirow{2}{*}{ Tree pruning (s) } & NoR-based & $<1$ & $<1$ \\
\cline { 2 - 4 } & Homogeneity-based & 28 & 2 \\
\hline \multirow{2}{*}{ Total (s) } & NoR-based & $<827$ & $<176$ \\
\cline { 2 - 4 } & Homogeneity-based & 854 & 177 \\
\hline
\end{tabular}

Table 1. The execution time of P-BPT and GSRM-BPT

Then, the pruning strategy based on region homogeneity is used, and the results are shown in Figure 6 and Figure 7. The homogeneity threshold in Figure 6 is $\delta_{p}=-0.5$, and NoR $=$ 16322. The homogeneity threshold in Figure 7 is $\delta_{p}=-4.5$, and NoR $=10807$. From Figure 6 and Figure 7, we can see that the results of P-BPT and GSRM-BPT are also similar. However, the consuming time of P-BPT pruning was $28 \mathrm{~s}$, and the GSRM-BPT pruning just consumed $2 \mathrm{~s}$.

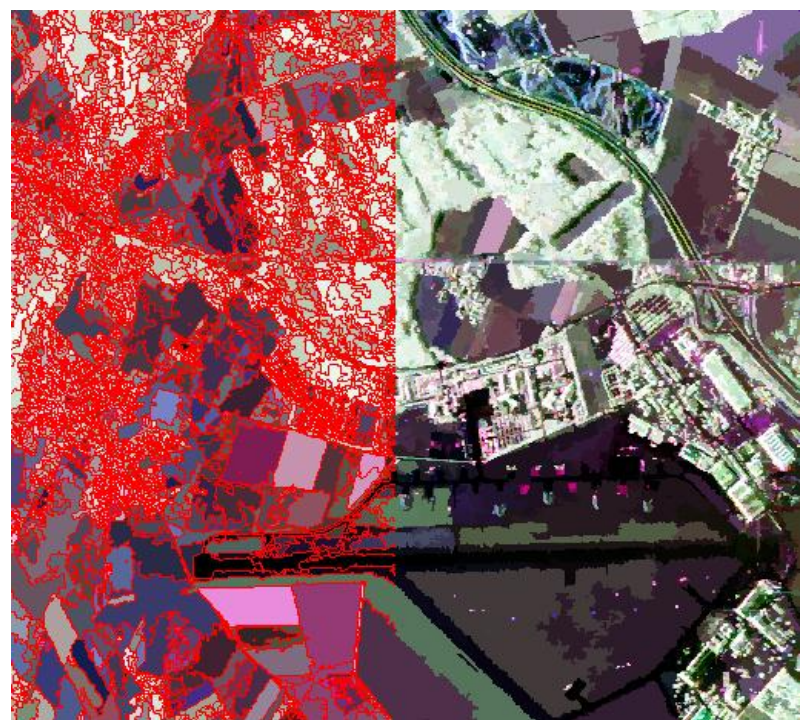

Figure 6. Segmentation result of P-BPT using the homogeneitybased pruning strategy

Compared with the NoR-based pruning method, the region sizes of the region-homogeneity-based pruning are very different: in homogeneous regions, the segmented regions are big, and in heterogeneous regions, the segmented regions are small. Since the NoR of the P-BPT result is far more than that of the GSRM$\mathrm{BPT}$ result, we can deduce that there are much more small regions in the P-BPT result than the GSRM-BPT result.

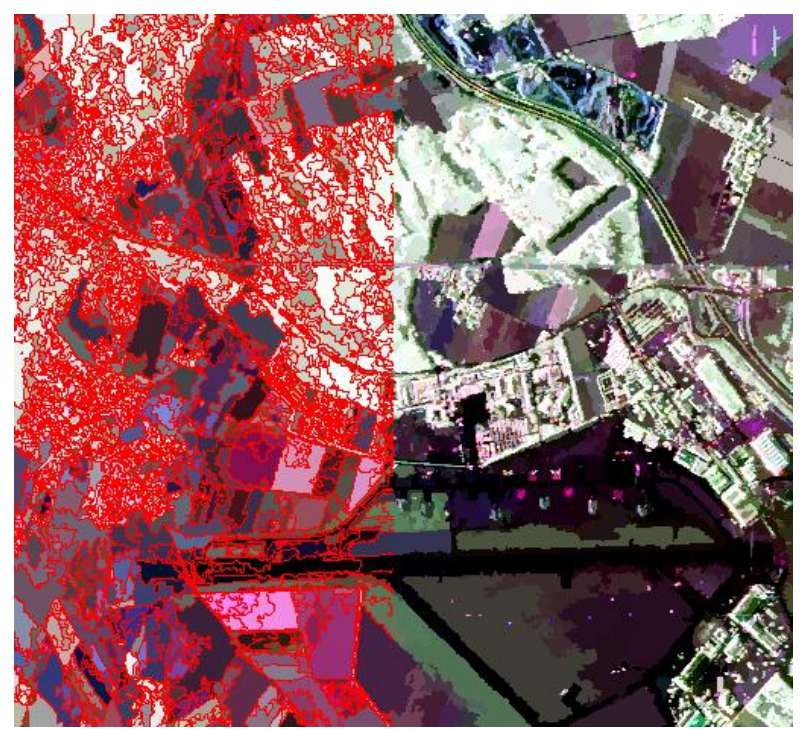

Figure 7. Segmentation result of GSRM-BPT using the homogeneity-based pruning strategy

The GSRM-BPT multiscale segmentation results using the NoR-based pruning strategy are shown in Figure 8, from which we can see that the less the segmented regions, the bigger the segmentation scale, the more the detail information is ignored, and the more obvious the outline information.

\section{CONCLUTIONS}

In this paper, we proposed a superpixel-based BPT segmentation algorithm by combining the GSRM algorithm and the BPT algorithm. Since the number of superpixels is much less than the number of pixels, the efficiency of the BPT algorithm is significantly improved. Meanwhile, the GSRM superpixel preserved the detail information in the PolSAR image very well. The proposed algorithm inherits the advantages of both GSRM and BPT. Experiments using the ESAR L-band image over the Oberpfaffenhofen test site proved the effectiveness of the proposed method.

\section{ACKNOWLEDGEMENTS}

The authors would like to thank the PolSARpro project distributed by ESA for providing the experimental data. This work is supported by "the Fundamental Research Funds for the Central Universities" (2015QNA29), and "A Project Funded by the Priority Academic Program Development of Jiangsu Higher Education Institutions" (PAPD).

\section{REFERENCES}

Alonso-Gonzalez A, Lopez-Martinez C, and Salembier P, 2010. Filtering and Segmentation of Polarimetric SAR Images with Binary Partition Trees. In: Proc. IGARSS 2010: 4043-4046.

Alonso-González A, López-Martínez C, and Salembier P, 2011. Binary Partition Tree as a Polarimetric SAR Data Representation in the Space-time Domain. In: Proc. IGARSS 2011: 3819-3822. 


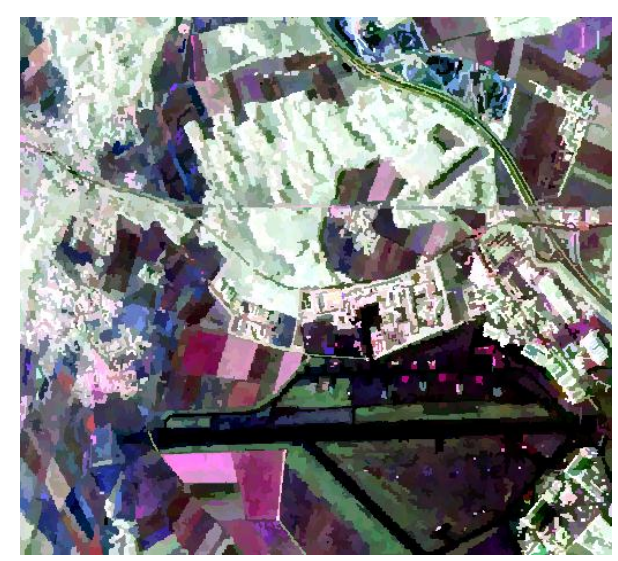

a. NoR $=4000$

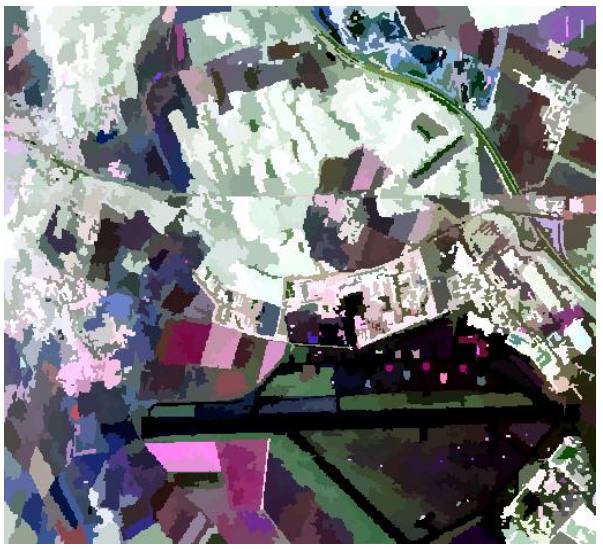

c. $\mathrm{NoR}=1000$

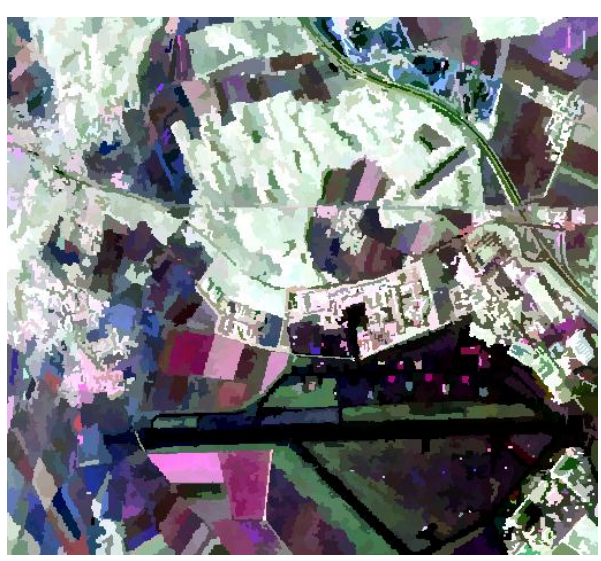

b. $\mathrm{NoR}=2000$

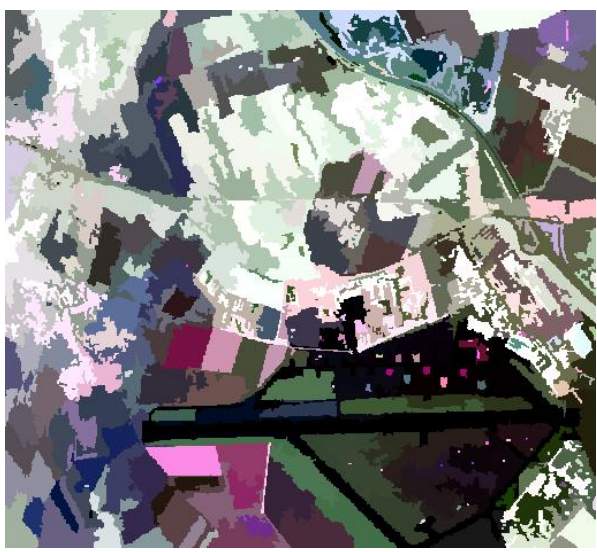

d. $\mathrm{NoR}=500$

Figrue 8. The GSRM-BPT multiscale segmentation results using the NoR-based pruning strategy

Alonso-González A, López-Martínez C, and Salembier P, 2012. Filtering and Segmentation of Polarimetric SAR Data Based on Binary Partition Trees. IEEE Transactions on Geoscience and Remote Sensing, 50(2): 593-605.

Guigues L, Le Men H, and Cocquerez J-P, 2003. Scale-sets image analysis. In: Proc. International Conference on Image Processing: 45-48.

Jolion J-M, and Montanvert A, 1992. The adaptive pyramid, a framework for $2 \mathrm{~d}$ image analysis. In: Proc. CVGIF: Image Understanding, 55(3): 339-348.

Koepfler G, Lopez C, and Morel J. M, 1994. A Multiscale Algorithm for Image Segmentation by Variational Method. SIAM Journal on Numerical Analysis, 3(1): 282-299.

Koenderink J J, 1984. The structure of images. Biol. Cybern., 50: 363-370.

Lang F, Yang J, Zhao L, and Li D, 2012. Hierarchical Classification of Polarimetric SAR Image Based on Statistical Region Merging. In: ISPRS Ann. Photogramm. Remote Sens. Spatial Inf. Sci., I-7: 147-152.

Lang F, Yang J, Li D, et al, 2014. Polarimetric SAR Image Segmentation Using Statistical Region Merging. IEEE Geoscience and Remote Sensing Letters, 11(2): 509-513.

Li H T, Gu H Y, Han Y S, and Yang J H, 2008. Object-oriented classification of polarimetric SAR imagery based on Statistical Region Merging and Support Vector Machine. In: Proc.
International Workshop on Earth Observation and Remote Sensing Applications: 1-6.

Liu B, Hu H, Wang H, et al, 2013. Superpixel-Based Classification with an Adaptive Number of Classes for Polarimetric SAR Images. IEEE Transactions on Geoscience and Remote Sensing, 51(2): 907-24.

Nock R, and Nielsen F, 2004. Statistical Region Merging. IEEE Transactions on Pattern Analysis and Machine Intelligence, 26(11): 1452-1458.

Samet H, Webber R E, 1985. Storing a Collection of Polygons Using Quadtrees. ACM Transactions on Graphics, 4(3): 182222 .

Salembier P, and Garrido L, 2000. Binary partition tree as an efficient representation for image processing, segmentation, and information retrieval. IEEE Transactions on Image Processing, 9(4): 561-76.

Shi J, and Malik J, 2000. Normalized cuts and image segmentation. IEEE Transactions on Pattern Analysis and Machine Intelligence, 22(8): 888-905.

Witkin A W, 1983. Scale space filtering. In: Proc. of 8th Int. Joint Conf. on Artficial Intell., Karlsruhe, West Germany: 1019-1021. 